\title{
A Dispersive Optical Model Analysis of the Alpha Particles Scattering by Titanium Element Nucleus and Its Natural Isotopes
}

\author{
Haiddar Al-Mustafa, Anees Belal \\ Department of Physics, Faculty of Science, Al-Baath University, Homs, Syria \\ Email address: \\ HAIDDAR2018@gmail.com (H. Al-Mustafa),draneesblal@gmail.com (A. Belal)
}

\section{To cite this article:}

Haiddar Al-Mustafa, Anees Belal. A Dispersive Optical Model Analysis of the Alpha Particles Scattering by Titanium Element Nucleus and Its Natural Isotopes. Nuclear Science. Vol. 5, No. 1, 2020, pp. 8-15. doi: 10.11648/j.ns.20200501.12

Received: February 2, 2020; Accepted: March 9, 2020; Published: April 30, 2020

\begin{abstract}
A dispersive optical model analysis of the alpha particles scattering by titanium element nucleus and its natural isotopes has been applied for a new scattering potential within the energy range (1-100) MeV which has contained the range of the Coulomb barrier, and for constant input values of the parameters of this potential. This potential is extent of the mean field potential and is called by (coulomb-nuclear) interference potential, that contains (spin-orbit) coulomb term. The results according to DOMACNIP program that has been designed for that purpose would contain: continuous energy variation of the depths of the real and imaginary parts of the mean field, which are connected by dispersion relations have been compared with these resulting from global parameterizations of the alpha particles scattering potential. In addition to continuous energy variation of the real radius parameter of the Wood-Saxon approximation to the mean field potential with its Hatree-Fock approximation of the nonlocal potential. Consequently, our results for the continuous energy variations of the predicted total reaction cross section within the energy range (1-100) $\mathrm{MeV}$, and with calculation step of the pervious range whose magnitude $(1 \mathrm{MeV})$, differential cross sections, Ratio of the differential elastic scattering cross section to Rutherford cross section and polarization resulted only from the Coulomb spin-orbit term that has been appeared characteristically for selected energy and for selected center-of-mass scattering angle within the energy range (1-100) $\mathrm{MeV}$, showed the excellent agreement with available experimental data and better than these resulted from global parameterizations of the alpha particles scattering potential.
\end{abstract}

Keywords: Dispersive Optical Model Analysis (DOMA), Alpha Particles Scattering,

(Coulomb-Nuclear) Interference Potential (CNIP), Hatree-Fock potential (HF), Dispersion Relations (DR),

Cross Section, Polarization, Coulomb Barrier

\section{Introduction}

The interaction between a nucleus and a nucleus (even if one of them is a single nucleon), is one of the most important aspects in nuclear physics. It governs the elastic and inelastic scattering and determines the behavior of the various channels in nuclear reactions [1-3]. This interaction is a complicated many-body problem even for the analysis of elastic scattering. All the nucleons of the target nucleus interact with each other and with the incident nucleon, and each individual nucleon-nucleon interaction contains central exchange and tensor components. The optical model approximates of this complicated interaction by a two-body potential between the incident particle and the target nucleus. The detailed structure of the target nucleus and incident nucleus is ignored. The optical model takes that name as the scattering of nucleons by nuclei parallels the scattering of light waves by a refracting sphere ("cloudy" crystal ball). In that model the elastic scattering may be compared with refraction of optical waves and the inelastic scattering with absorption due to the fact that the crystal ball is cloudy [4].

The origin of the optical model potential is the average interaction between the nucleons in the projectile with those in the target nucleus. The scattering is represented in terms of a complex potential $U$, [5-9]: 


$$
U(r, E)=-V_{V}(r, E)-V_{S O}(r, E) \cdot \vec{\sigma} \cdot \vec{l}+V_{S}(r, E)+V_{C}(r)+i\left(-W_{V}(r, E)-W_{S}(r, E)+W_{S O}(r, E) \cdot \vec{\sigma} \cdot \vec{l}\right)
$$

Where $V_{V, S}$ and $W_{V, S, S O}$ are the real and imaginary components of the volume-central $(V)$, surface-central $(S)$ and spin-orbit $(S O)$ potentials, respectively. $E$ is the LAB energy of the incident particle in $\mathrm{MeV}$. All components are separated in energy-dependent well depths, $V_{V}, V_{S}, W_{V}, W_{S}$ and $W_{S O}$, and energy-independent radial parts $f$, namely

$$
\begin{gathered}
V_{V}(r, E)=V_{V}(E) f\left(r, R_{V}, a_{V}\right) \\
W_{V}(r, E)=W_{V}(E) f\left(r, R_{V}, a_{V}\right) \\
W_{S}(r, E)=-4 a_{S} W_{S}(E) \frac{d}{d r} f\left(r, R_{S}, a_{S}\right) \\
V_{S}(r, E)=-4 a_{S} V_{S}(E) \frac{d}{d r} f\left(r, R_{S}, a_{S}\right) \\
V_{S O}(r, E)=V_{S O}(E)\left(\frac{\hbar}{m_{\pi} c}\right)^{2} \frac{1 d}{r d r} f\left(r, R_{S O}, a_{S O}\right) \\
W_{S O}(r, E)=W_{S O}(E)\left(\frac{\hbar}{m_{\pi} c}\right)^{2} \frac{1 d}{r d r} f\left(r, R_{S O}, a_{S O}\right)
\end{gathered}
$$

The form factor $f\left(r, R_{i}, a_{i}\right)$ is a Wood-Saxon shape

$$
f\left(r, R_{i}, a_{i}\right)=\frac{1}{\left[1+e^{\left(\frac{r-R_{i}}{a_{i}}\right)}\right]}
$$

Where the geometry parameters are the radius $R_{i}=r_{i} A^{\frac{1}{3}}$, with $A$ the atomic mass number, and the diffuseness parameters $a_{i}, i=V, S O, S$. For charged particles, the coulomb term $V_{C}$, as usual, is given by that of a uniformly charged sphere,

$$
\begin{gathered}
V_{C}(r)=\left(Z Z^{\prime} e^{2} / 2 R_{C}\right)\left(3-r^{2} / R_{C}^{2}\right): r \leq R_{C} \\
=Z Z^{\prime} e^{2} / r: r \geq R_{C}
\end{gathered}
$$

With $Z\left(Z^{\prime}\right)$ the charge of the target (projectile), and $R_{C}=r_{C} A^{\frac{1}{3}}$ the Coulomb radius.

In addition of the term $U_{C, S O}$, the Coulomb spin-orbit term that is assumed to have the form:

$$
U_{C, S O}=\left(\mu_{P}-\frac{1}{2}\right) \frac{1}{M_{p}^{2} c^{2}}\left[\frac{1}{r} \frac{d}{d r} V_{C}(r)\right] \vec{\sigma} \cdot \vec{l}
$$

Where $\mu_{P}$ is the proton magnetic moment in nuclear magnetons.

We have got the (Coulomb-Nuclear) interference potential which is expressed as follows:

$$
U_{C N I P}(r, E)=V_{o p t}(r, E)+i W_{o p t}(r, E)
$$

Where:

$$
\left\{\begin{array}{c}
V_{o p t}(r, E)=U_{C}(r)+U_{S}(r, E)-U_{V}(r, E)+ \\
\left\{-U_{S O}(r, E)+U_{C, S O}(r)\right\}\left(\begin{array}{c}
l \\
o r \\
-l-1
\end{array}\right) \\
W_{o p t}(r, E)=-W_{V}(r, E)-W_{S}(r, E)+W_{S O}(r, E)\left(\begin{array}{c}
l \\
o r \\
-l-1
\end{array}\right)
\end{array}\right.
$$

Here, $l$ for $j=l+1 / 2$ and $-l-1$ for $j=l-1 / 2$

By solving the Schrödinger equation numerically with this complex potential yields a wealth of valuable information; it returns a prediction for the basic observables, namely the elastic angular distribution and polarization, the total reaction cross section, and the detailed information of the calculation methodology with this potential in addition to M3Y interaction according to the folding models are showed in the reference [9].

It is of interest to determine the (Coulomb-Nuclear) interference potential of the alpha-nucleus scattering as knowledge that is important for understanding many nuclear and astrophysical processes, involving alpha particles in the entrance and the exit channels.

The dispersive optical model analysis describes the continuous energy variation of the nuclear (Coulomb-Nuclear) interference potential components depths and connection between the real parts and imaginary parts of this potential by a dispersion relation, and so the reliable determination of this potential is perfect by comparing a prediction of the cross sections and elastic angular distribution with these measured experimentally.

There are many published studies for detailed analyses of data for the obtaining global alpha - nucleus potentials (in both phenomenological and microscopic states of the potential) which are valid for fitting of the experimental data. Therefore, our dependence is on global parametrizations of the alpha scattering potential which agree with the energy and atomic mass ranges of the titanium element nucleus and its natural isotopes.

The present paper aims at presenting the dispersive optical model analysis (DOMA) of the alpha scattering by titanium element nucleus and its natural isotopes by using a complex (Coulomb-Nuclear) interference potential and comparing the results with these resulted from global parametrizations of the optical model potential and available experimental data within energy range (1-100) $\mathrm{MeV}$ and with calculation step of the previous range whose magnitude $1 \mathrm{MeV}$.

\section{Methodology}

The methodology of a dispersive optical model analysis is similar to the proton scattering that is showed in references $[10,11]$, but in state of alpha particles scattering by titanium element nucleus and its natural isotopes within the energy range (1-100) MeV shows clearly influence of the behavior, known as potential threshold anomaly, in addition to an appearance of clearly influence of the Coulomb spin-orbit term and this feature is new in such researches. Therefore, in this paper, we have depended on the following methodology:

i. Replacing the symbol $E_{f}$ (Fermi energy) in Brown-Rho expression with the symbol $E_{S}$ (reference energy) for a complex and a heavy particles scattering.

ii. The values of the input parameters have been constant for titanium element nucleus and its natural isotopes, as they are showed in the table 1 .

iii. Comparing the continuous energy variation of the (Coulomb-Nuclear) interference potential components depths and the resulted cross sections, elastic angular distribution and polarization with global parametrizations of the alpha scattering potential and available experimental data as follows:

After calculating the depths, the geometrical parameters 
and the volume integral per nucleon of the alpha scattering potential components, we have compared them with global parameterizations of the alpha particles potential whose calculations have been performed in the DOMACNIP program:

1. Avrigeanu et al [12], its coding in the program Avr, for

$$
\mathrm{E}<50 \mathrm{MeV}, \mathrm{Z}_{\mathrm{t}}=(21-83), \mathrm{A}_{\mathrm{t}}=(45-209)
$$

2. Koning and Delaroche [5], its coding in the program $\mathrm{KD}$, for

$$
0.001 \leq \mathrm{E} \leq 200 \mathrm{MeV}, \mathrm{Z}_{\mathrm{t}}=(12-83), \mathrm{A}_{\mathrm{t}}=(24-209)
$$

While the values of a spin-orbit coupling term depths of the alpha particles scattering potential in our calculations are:

$\mathrm{V}_{\mathrm{SO}}=\mathrm{V}_{\mathrm{SO}_{\mathrm{KD}}}=\mathrm{V}_{\mathrm{SO}_{\mathrm{Avr}}}=0, \mathrm{~W}_{\mathrm{SO}}=\mathrm{W}_{\mathrm{SO}_{\mathrm{KD}}}=\mathrm{W}_{\mathrm{SO}_{\mathrm{Avr}}}=0$

Therefore, the polarization yields only from the Coulomb spin-orbit term.

Also, for the value of coulomb radius parameter, $r_{C}=$ $\mathrm{r}_{\mathrm{KD}}$

Also, we have compared our results with these global parameterizations for the continuous energy variations of the predicted total reaction cross section, elastic angular distribution, and polarization for selected center-of-mass scattering angle within the energy range (1-100) $\mathrm{MeV}$ and with calculation step of the pervious range whose magnitude $(1 \mathrm{MeV})$, in addition to elastic angular distribution and polarization for the energy value $(16 \mathrm{MeV})$ and within the angular range of the center-of-mass scattering angle $\left(5^{\circ}-\right.$ $175^{\circ}$ ) and with calculation step of the pervious range whose magnitude $\left(5^{\circ}\right)$.

\section{Results and Discussion}

The results According to DOMACNIP program are summarized as follows:

\subsection{Input Parameters}

The values of the constant input parameters for the alpha particles scattering by titanium element nucleus and its natural isotopes are showed in the (Table 1).

\begin{tabular}{|c|c|c|c|c|c|c|c|}
\hline \multicolumn{8}{|c|}{ Constant Input Parameters } \\
\hline \multicolumn{4}{|c|}{ Brown-Rho Parameters } & \multicolumn{4}{|c|}{ Geometrical Parameters Volume and Surface Absorption } \\
\hline$\rho_{\mathrm{w}}, \mathrm{MeV}$ & $\rho_{\mathrm{W}_{\mathrm{V}}}, \mathrm{MeV}$ & $\beta$, MeV.fm ${ }^{3}$ & $\mathrm{E}_{\mathrm{S}}(\mathrm{MeV})$ & $\mathbf{r}_{\mathrm{wv}}, \mathbf{f m}$ & $\mathbf{r}_{\mathrm{ws}}, \mathbf{f m}$ & $a_{w v}, f m$ & $\mathbf{a}_{\mathrm{ws}}, \mathbf{f m}$ \\
\hline 2.1 & 14.9 & -100.3 & -1.13 & 1.27 & 1.2 & 0.68 & 0.52 \\
\hline
\end{tabular}

Table 1. The input values of the alpha particles scattering potential parameters.

\begin{tabular}{lllll}
\hline Hartree-Fock Parameters & & & \multicolumn{2}{c}{ Diffuseness Parameter } \\
\hline $\mathbf{r}_{\mathrm{HF}}, \mathbf{f m}$ & $\mathbf{a}_{\mathrm{HF}}, \mathbf{f m}$ & $\boldsymbol{\alpha}_{\mathrm{HF}}$ & $\boldsymbol{V}_{\mathrm{HF}}\left(\mathbf{E}_{\mathbf{S}}\right), \mathbf{M e V}$ & $\mathbf{a}_{\mathbf{V}}, \mathbf{f m}$ \\
\hline 1.43 & 0.72 & 0.72 & -140.8 & 0.7 \\
\hline
\end{tabular}

\begin{tabular}{|c|c|c|c|c|c|}
\hline \multicolumn{6}{|l|}{ Changed Input Parameters } \\
\hline \multirow{2}{*}{ Titanium nucleus Ti (Na) } & \multicolumn{5}{|c|}{ Natural Titanium Isotopes } \\
\hline & $\mathrm{Ti}-46$ & $\mathbf{T i}-47$ & $\mathrm{Ti}-48$ & $\mathrm{Ti}-49$ & $\mathbf{T i}-\mathbf{5 0}$ \\
\hline$A_{t}(a m u)[13-16]$ & 45.9526 & 46.9518 & 47.948 & 48.9479 & 49.9448 \\
\hline Relative Abundance [15-16] & $8.25 \%$ & $7.44 \%$ & $73.72 \%$ & $5.41 \%$ & $5.18 \%$ \\
\hline
\end{tabular}

\begin{tabular}{llll}
\hline (Projectile-Target) Parameters & & Maximum Energy \\
\hline $\mathbf{Z}_{\mathbf{P}}$ & $\mathbf{A}_{\mathbf{P}}(\mathbf{a m u})$ & $\mathbf{Z}_{\mathbf{t}}$ & $\mathbf{E}_{\text {Lab }}(\mathbf{M a x}) \mathbf{M e V}$ \\
\hline 2 & 4.0026 & 22 & 100 \\
\hline
\end{tabular}

\subsection{Depths of the Alpha Particles Scattering Potential}

The depths of the real and imaginary (volume and surface) parts of the alpha particles scattering potential are compared with these resulted from global parameterizations of the optical potential within the energy range $(1-100) \mathrm{MeV}$ and with calculation step of the previous range whose magnitude $1 \mathrm{MeV}$, as they are showed in the Figures 1-3. From the figures it becomes clear for us: At energies by near Coulomb barrier a rapid variation of the depths of the real and imaginary (volume and surface) parts of the alpha potential around the Coulomb barrier is observed and visualized as a localized peak in the strength of the real potential, associated with a sharp decrease in the strength of the imaginary potential as it becomes more and more unimportant to remove flux from the reaction in this low energy region. This behavior, known as potential threshold anomaly, is attributed to a strong coupling between the elastic and the other channels. As the collision energy is lowered, reaction channels such as inelastic scattering, nucleon or cluster transfer, fusion and fission become effectively less important. 

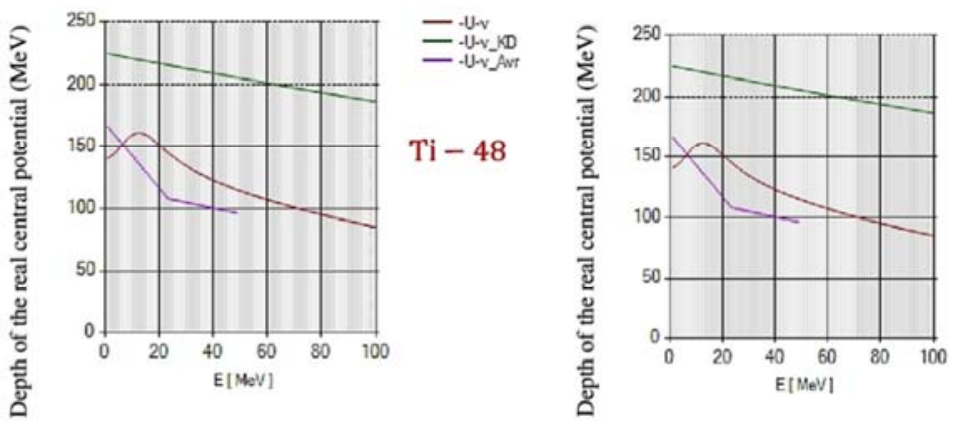

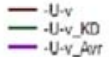

$\mathrm{Ti}(\mathrm{Na})$

Figure 1. Depth of the real part of the alpha particles scattering potential as a function of alpha energy (the red line) compared with these resulted from global parametrization of the optical model potential.
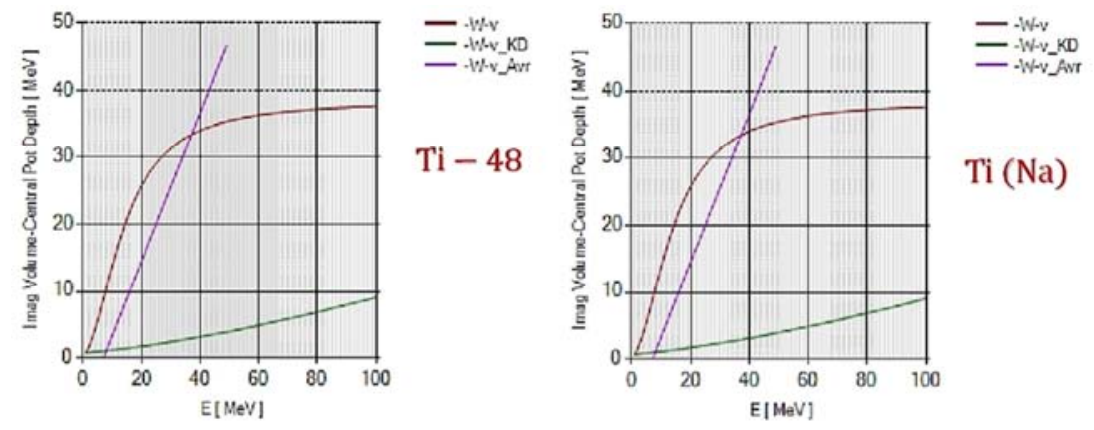

Figure 2. Depth of the volume component of the imaginary part of the alpha particles scattering potential as a function of alpha energy (the red line) compared with these resulted from global parametrization of the optical model potential.
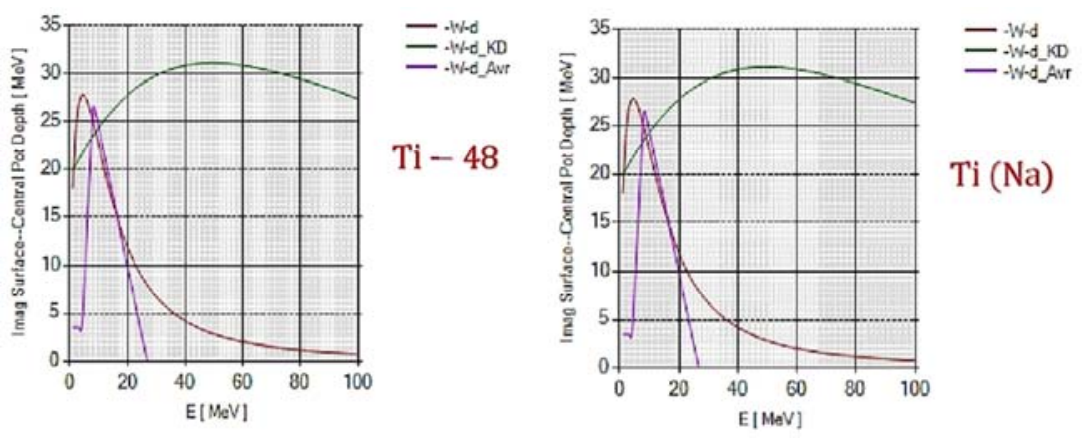

Figure 3. Depth of the surface-peaked component of the imaginary part of the alpha particles scattering potential as a function of alpha energy (the red line) compared with these resulted from global parametrization of the optical model potential.

\subsection{The Real Radius Parameter of the Alpha Particles Scattering Potential}

The real radius parameter of the Wood-Saxon approximation to the mean field potential with its $\mathrm{HF}$ approximation for alpha particles scattering, within the

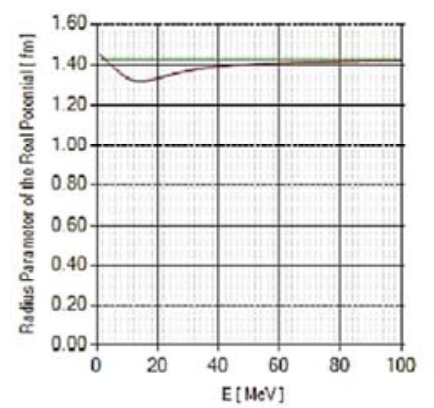

energy range $\left(E_{S}-100\right) \mathrm{MeV}$ and with calculation step of the previous range whose magnitude $1 \mathrm{MeV}$, as it is showed in the Figure 4, which appears as a rapid variation of the real radius parameter around the Coulomb barrier.



Figure 4. The energy dependence of the real radius parameter of the Wood-Saxon approximation to the mean field potential for alpha particles scattering with its HF approximation. 


\subsection{Cross Sections}

The total reaction cross sections within the energy range $(1-100) \mathrm{MeV}$ and with calculation step of the pervious range whose magnitude $1 \mathrm{MeV}$ are compared with these resulted from global parameterizations of the optical
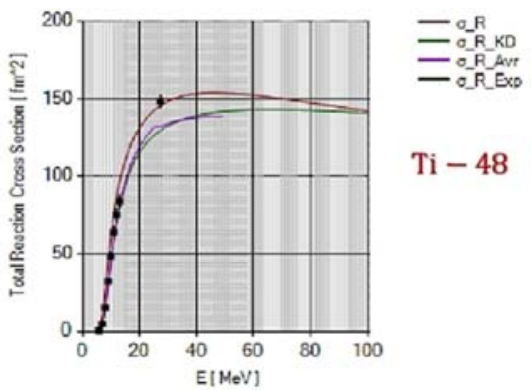

potential and with available experimental data [17, 18], and are in $\left(\mathrm{fm}^{2}=10 \mathrm{mb}\right)$, as it is showed in the Figure 5 . There is excellent agreement with the experimental data and better than these resulted from the global parametrization of the optical potential according to our calculations.

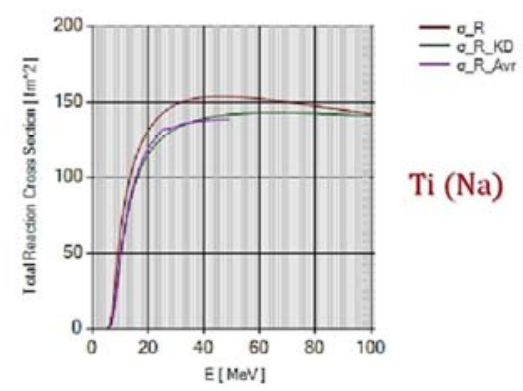

Figure 5. The energy dependence of the $\left(\alpha+{ }^{(48, n a t u r a l)}\right.$ Ti $)$ total reaction cross section (the red line) compared with experimental values and with these resulted from the global parametrization of the optical model potential.

\subsection{Differential Cross Sections and Polarization for Selected Energy}

The differential cross sections and the polarization for selected energy whose magnitude (16) MeV compared with these resulted from global parameterizations of the optical potential, as which are showed in the Figures 6-9. According to our calculations: there is an excellent agreement with these resulted from global parametrizations of the optical model potential for differential cross sections, whereas the polarization which yielded only from the Coulomb spin-orbit term has been showed characteristically because the depths of spin-orbit term in the standard optical model potential equal zero $\left(\mathrm{V}_{\mathrm{SO}}=\right.$ $\mathrm{V}_{\mathrm{SO}_{\mathrm{KD}}}=\mathrm{V}_{\mathrm{SO}_{\mathrm{Avr}}}=0, \mathrm{~W}_{\mathrm{SO}}=\mathrm{W}_{\mathrm{SO}_{\mathrm{KD}}}=\mathrm{W}_{\mathrm{SO}_{\mathrm{Avr}}}=0$ ).

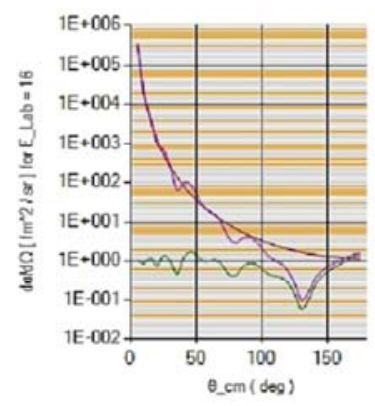

\subsection{Differential Cross Sections and Polarization for Selected Angle}

The differential cross sections and polarization for selected angle whose magnitude $\theta_{\mathrm{cm}}=30^{\circ}$ compared with these resulted from global parameterizations of the optical potential, as which are showed in the Figures 10-13. According to our calculations: there is good agreement with a simple deviation compared with these resulted from global parametrizations of the optical model potential for differential cross sections whereas the polarization which yielded only from the Coulomb spin-orbit term has been showed characteristically.

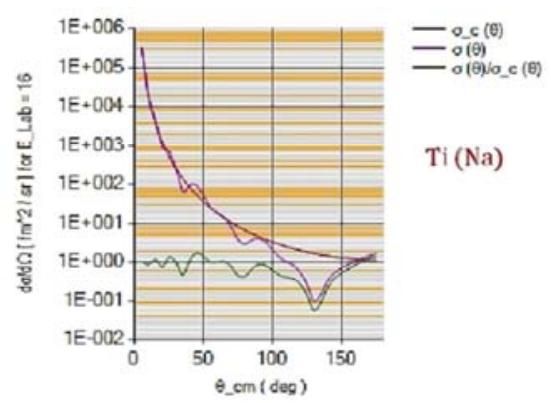

Figure 6. Dependence of the $\left(\alpha+{ }^{(48, n a t u r a l)}\right.$ Ti $)$ Coulomb, elastic differential cross sections and $\left(\sigma_{C} / \sigma\right)$ ratio upon the center-of-mass scattering angle, for $E_{\text {Lab }}=16 \mathrm{MeV}$.
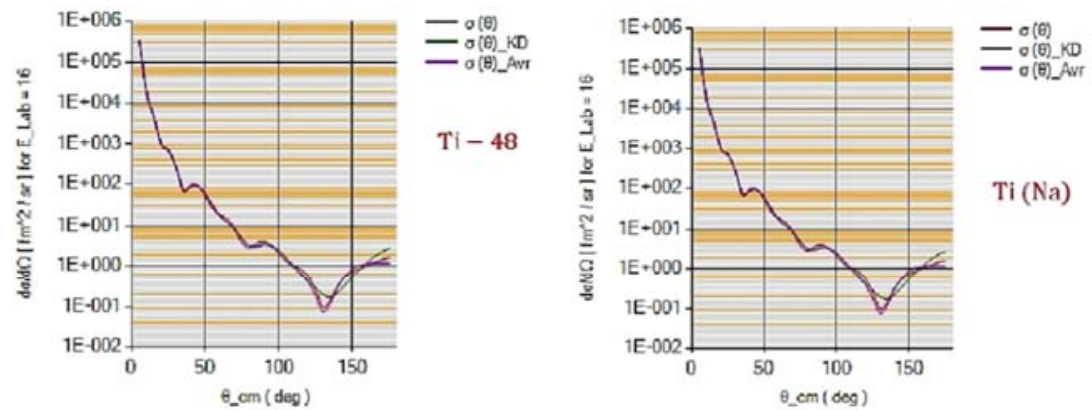

Figure 7. Dependence of the $\left.\left(\alpha+{ }^{(48, n a t u r a l}\right) T i\right)$ elastic differential cross section upon the center-of-mass scattering angle (the red line) compared with these resulted from global parametrization of the optical model potential, for $E_{L a b}=16 \mathrm{MeV}$. 

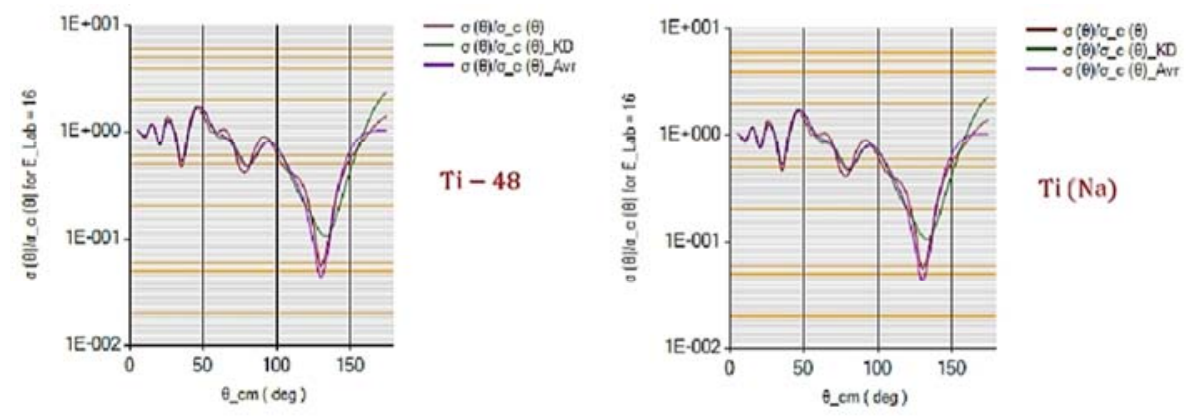

Figure 8. Dependence of the $\left.\left(\alpha+{ }^{(48, n a t u r a l}\right) T i\right)\left(\sigma_{C} / \sigma\right)$ ratio upon the center-of-mass scattering angle (the red line) compared with these resulted from global parametrization of the optical model potential, for $E_{L a b}=16 \mathrm{MeV}$.
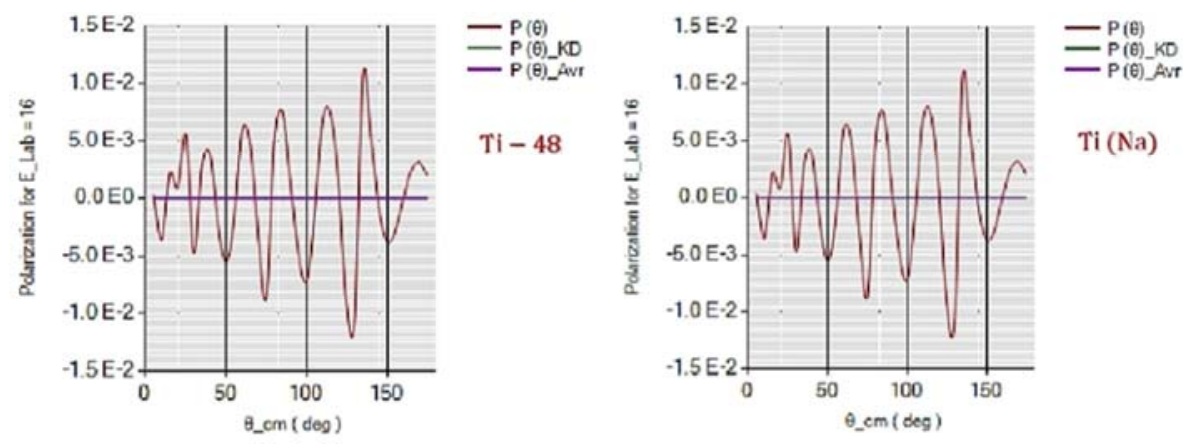

Figure 9. Dependence of the $\left(\alpha+{ }^{(48, n a t u r a l)}\right.$ Ti) polarization upon the center-of-mass scattering angle (the red line) compared with these resulted from global parametrization of the optical model potential, for $E_{\text {Lab }}=16 \mathrm{MeV}$.
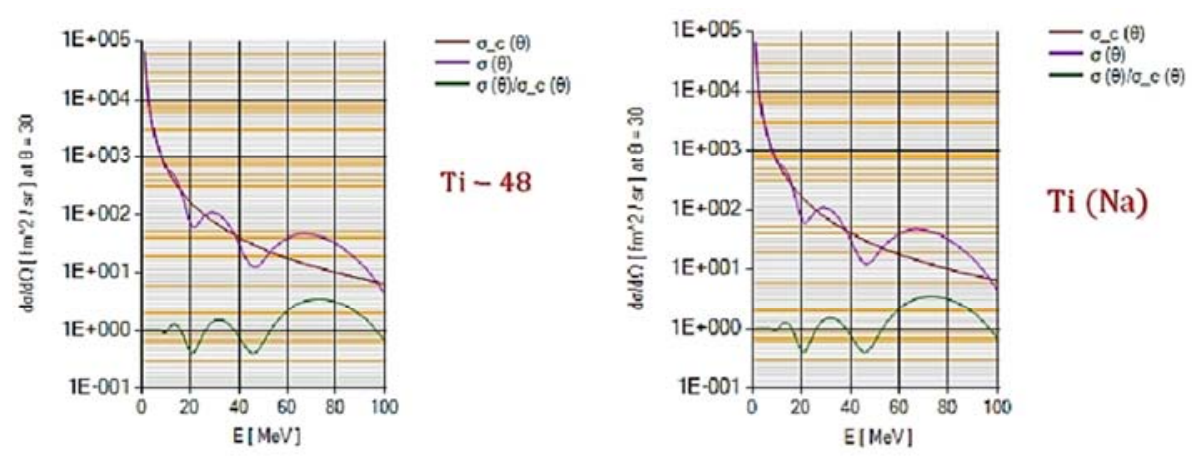

Figure 10. The energy dependence of the $\left(\alpha+{ }^{(48, n a t u r a l)} T i\right)$ Coulomb, elastic differential cross sections and $\left(\sigma_{C} / \sigma\right)$ ratio for the center-of-mass scattering angle, $\theta_{c m}=30^{\circ}$.
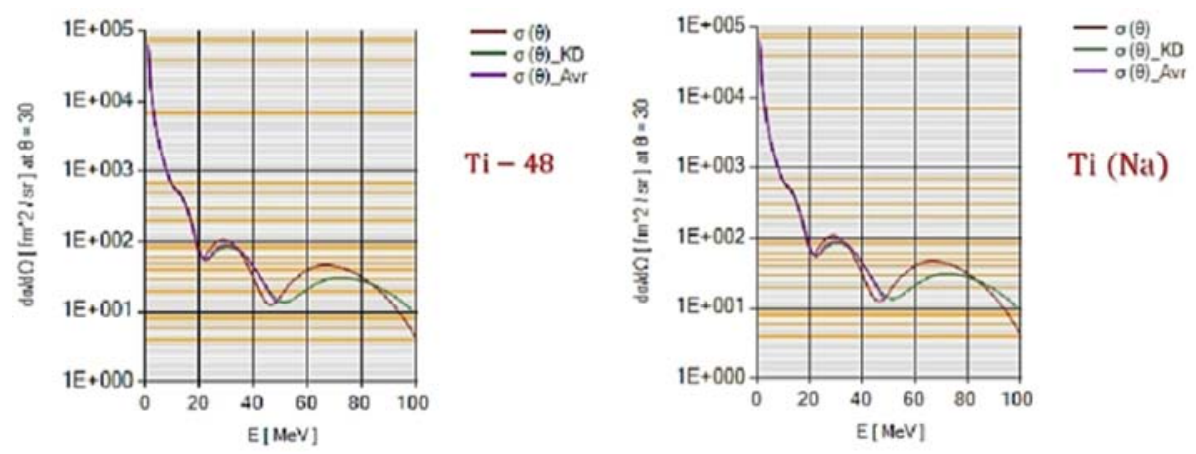

Figure 11. The energy dependence of the $\left(\alpha+{ }^{(48, n a t u r a l)} T i\right)$ elastic differential cross section (the red line) compared with these resulted from global parametrization of the optical model potential, for $\theta_{c m}=30^{\circ}$. 

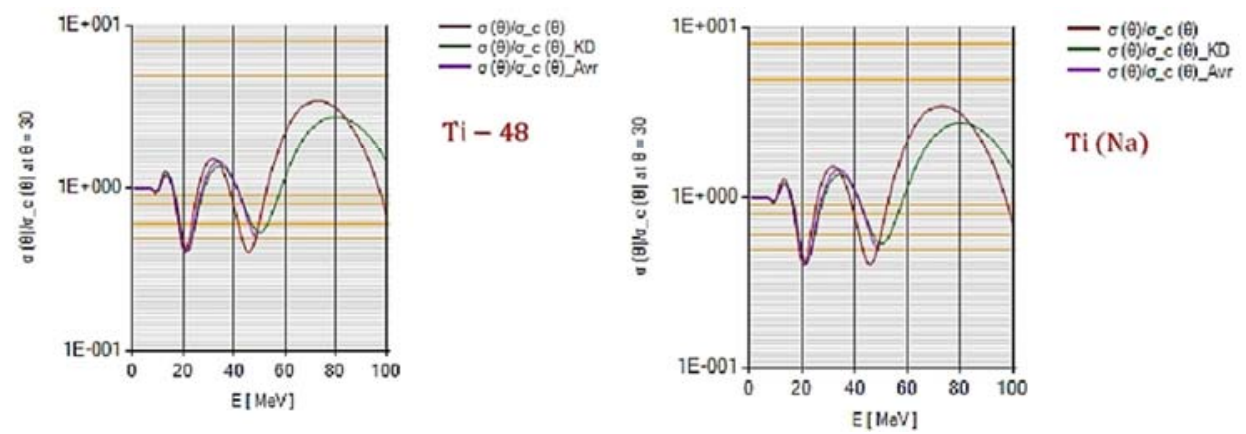

Figure 12. The energy dependence of the $\left(\alpha+{ }^{(48, n a t u r a l)} \mathrm{Ti}\right)\left(\sigma_{C} / \sigma\right)$ ratio (the red line) compared with these resulted from global parametrization of the optical model potential, for $\theta_{c m}=30^{\circ}$.


Figure 13. The energy dependence of the $\left(\alpha+{ }^{(48, n a t u r a l)} \mathrm{Ti}\right)$ polarization (the red line) compared with these resulted from global parametrization of the optical model potential, for $\theta_{c m}=30^{\circ}$.

\section{Conclusion}

The important conclusions can be shown as follows:

i. Our result according to the dispersive optical model analysis of the alpha particles scattering by titanium element nucleus and its natural isotopes have been drawn for constant input value of the (coulomb-nuclear) interference potential parameters.

ii. Our calculation within the energy range $(1-100) \mathrm{MeV}$ and with calculation step of the pervious range whose magnitude $1 \mathrm{MeV}$ of the continuous energy variation of the depths of the real and imaginary parts of the alpha particles scattering potential were compared with these resulting from global parameterization of the optical model potential, in addition to continuous energy variation of the real radius parameter of the WoodSaxon approximation to the mean field potential with its Hatree-Fock approximation of the nonlocal potential for alpha particles scattering within the energy range $\left(E_{S}-100\right) \mathrm{MeV}$ have been showed clearly influence of the potential threshold anomaly behavior.

iii. Our prediction of the total reaction cross section data within the energy range $(1-100) \mathrm{MeV}$ showed excellent agreement with available experimental data and the better than these resulted from global parameterization of the optical model potential.

iv. Our prediction of the differential cross section data for selected energy $(16 \mathrm{MeV})$ within the angular range $\theta_{c m}=\left(5^{\circ}-175^{\circ}\right)$, showed excellent agreement with these resulted from global parameterization of the optical model potential and good agreement with a simple deviation for selected angle $\theta_{c m}=30^{\circ}$ within the energy range $(1-100) \mathrm{MeV}$ compared with these resulting from global parameterization of the optical model potential. and thus more reliable for calculation the cross sections of unknown interactions of elements nuclei and their isotopes such alpha particles scattering by titanium element nucleus and its natural isotopes.

v. Our prediction of the polarization data resulting only from the Coulomb spin-orbit term for selected energy $(16 \mathrm{MeV})$ within the angular range $\theta_{c m}=\left(5^{\circ}-175^{\circ}\right)$, and also for selected angle $\theta_{c m}=30^{\circ}$ within the energy range $(1-100) \mathrm{MeV}$, has been appeared characteristically. This feature is new in such researches, which shows clearly influence of Coulomb spin-orbit term with increasing of alpha particles energy.

Depending upon these conclusions, the new potential ((coulomb-nuclear) interference potential) after adding the (spin-orbit) coulomb term contributed in analyzing and predicting experimental data and more accurately, and so explaining the behavior called threshold anomaly around the Coulomb barrier (rapid variation in the real and imaginary potential depths), which is attributed to a strong coupling between the elastic and the other channels. Couplings of these reaction channels may produce polarization potentials of different signs, attractive or repulsive, that dynamically decrease or increase the Coulomb barrier and especially for the complex and heavy charged particles scattering. 


\section{References}

[1] Hodgson, P. E. (1990). The unification of the nuclear optical potential, Contemporary Physics, 31: 5, 295-308, DOI: 10.1080/00107519008213780.

[2] Brandan, M. E., \& Satchler, G. R. (1997). Phys. Reports 285, 143-243.

[3] Alamanos, N., \& Roussel-Chomaz, P. (1996). Ann. Phys. Fr. 21, 601-668.

[4] Satchler, G. R. Introduction to Nuclear Reactions, second edition, ISBN 0333523792.

[5] Koning, A. J., \& Delaroche, J. P. (2003). Nucl. Phys. A713, 231.

[6] Mahaux, C., \& Sartor, R. (1991). Dispersion Relation Approach to the Mean Field and Spectral Functions of Nucleons in 40Ca, Nuclear Physics, A528, pp. 253-297, Elsevier Science Publishers B. V. (North-Holland).

[7] IAEA, (2006). Handbook for Calculations of Nuclear Reaction Data, RIPL-2, IAEA in Austria, (Final report of a coordinated research project, IAEA-TECDOC-1506), pp. 4769.

[8] Melkanoff, M. A, Saxon, D. S, Jnodvik, J. S., \& Cantor, D. G. (1961). A Fortran Program for Elastic Scattering Analyses with the Nuclear Optical Model, University of California Press Berkeley and Los Angeles, Retrieved August 24, 2009 [EBook \#29784], online at www.gutenberg.org, p. 111.

[9] Al-Mustafa, H., \& Belal. A. (2019). Program Design for Analyzing the Optical Model of the (Coulomb - Nuclear) Interference Potential, Journal of AL Baath University, HomsSyria, 41 (18), 71-102.

[10] Al-Mustafa, H., \& Belal. A. (2019). Program Design for
Analyzing the Dispersive Optical Model of the (Coulomb Nuclear) Interference Potential, Journal of AL Baath University, Homs- Syria, 41 (17), 51-80.

[11] Al-Mustafa, H., \& Belal. A. (2019). A Dispersive Optical Model Analysis of the Protons Scattering by Titanium Element Nucleus and Its Natural Isotopes, Nuclear Science, Science PG, 4 (4): 44-51, DOI: 10.11648/j.ns.20190404.12.

[12] Avrigeanu, M., \& Avrigeanu, V. (2010). Phys. Rev. C 82, 014606.

[13] Audi, G., \& Wapstra, A. H. (1993). The Isotopic Mass Data. Nucl. Phys A. 565, 1-65.

[14] Audi, G., \& Wapstra, A. H. (1995). The Isotopic Mass Data. Nucl. Phys A. 595, 409-480.

[15] Rosman, K. J. R., \& Taylor, P. D. P. (1999). The Percent Natural Abundance Data. (1997 report of the IUPAC Subcommittee for Isotopic Abundance Measurements). Pure Appl. Chem., 71, 1593-1607.

[16] Wieser, M. E. (2006). Atomic Weights of the Elements 2005. Department of Physics and Astronomy, University of Calgary, Calgary, Canada. (2006 IUPAC TECHNICAL REPORT). Pure Appl. Chem., Vol. 78, No. 11, pp. 2051-2066. DOI: 10.1351/pac200678112051.

[17] Vonach, H, Haight, R. C., \& Winkler, G. (1983). (Alpha, n) and Total Alpha-Reaction Cross Sections for Ti-48 and V-51. Physics Review, Part C, Nuclear Physics, volume 28, page 2278. (JANIS 4.0- Local- Incident alpha data / EXFOR / (Ti48) / (, NON) / (C0318.004) (8pts)).

[18] Bilaniuk, M. P, Tokarevskii, V. V, Bulkin, V. S, Dubar, L. V, Nemets, O. F., \& Slyusarenko, L. I, (1981). Deuteron and Alpha-Particle Total Reaction Cross Sections for Nuclei with A-50. Jour. Of Physics, Part G, (Nucl. and Part. Phys), volume 7, page 1699. (JANIS 4.0- Local- Incident Alpha data / EXFOR / (Ti48) / (, NON / (D5003.003) (1pts)). 